\title{
Reproducibility of cardiorespiratory measurements during submaximal and maximal running in children
}

\author{
V. B. Unnithan, L. A. Murray, J. A. Timmons, D. Buchanan* and J. Y. Paton* \\ Department of Child Health and *Statistics, University of Glasgow, Glasgow, UK.
}

\begin{abstract}
With the increased use of oxygen consumption measurements in clinical and sporting studies, measurement variability has become more important to both the paediatric clinician and the sports scientist. In this study we assessed the reproducibility of cardiorespiratory measurements during submaximal and maximal running in children. Ten healthy, physically active boys (mean(s.d.) age 10.7(0.71) years) performed two submaximal and two maximal running tests within a 4 week period. The submaximal protocol consisted of three 6 minute runs at $7.2,8.0$ and $8.8 \mathrm{~km} / \mathrm{h}$. Every attempt was made to minimize the sources of non-biological variability at each testing session. During submaximal exercise, oxygen consumption $\left(\mathrm{VO}_{2}\right)$, heart rate (HR) and fractional utilization appeared to be the most reliable measures accounting for over two-thirds of the total variation (coefficients of reliability (CR) of $68 \%, 94 \%$ and $82 \%$ respectively). Ventilation (Ve) and respiratory exchange ratio (RER) proved to be only moderately reliable accounting for less than half of the total variation (CR $50 \%$ and $45 \%$ respectively). At maximal exercise, $\mathrm{VO}_{2}, \mathrm{Ve}$ and time to exhaustion were most reliable, accounting for approximately two-thirds of the total variation (CR $65 \%, 63 \%$ and $63 \%$ respectively). Within this test environment, a two visit submaximal assessment was capable of estimating $\mathrm{VO}_{2}$ with a standard error of $\pm 1.25 \mathrm{ml} / \mathrm{kg} / \mathrm{min}$. Similarly, for maximal testing a two visit assessment estimated peak $\mathrm{VO}_{2}$ with a standard error of $\pm 2.28 \mathrm{ml} / \mathrm{kg} / \mathrm{min}$. On the evidence of these results a two visit assessment for submaximal and maximal exercise testing seems adequate to estimate the stability of submaximal cardiorespiratory measures and peak $\mathrm{VO}_{2}$ in healthy, normally active boys.
\end{abstract}

Keywords: paediatric, reproducibility, submaximal, maximal

The variability of cardiorespiratory measurements at submaximal and maximal exercise intensities is of considerable importance both to the paediatric sports scientist and to the paediatrician. Intervention, rehabilitation and training strategies are based on data from physiological testing. Knowledge of the level and natural variability both between and within individuals is critical to an understanding of the efficacy of a treatment or the presence of a training effect.

Address for correspondence: Dr V. B. Unnithan, Children's Exercise and Nutrition Centre, Chedoke Hospital, Evel Building (4th Floor), PO Box 2000, Station A, Hamilton, Ontario, Canada, L8N $3 Z 5$
The variability of submaximal exercise performance in adults has been investigated in a number of studies $^{1-5}$. These studies have found significant differences in the submaximal energy cost of running both between and within moderately and welltrained runners on repeated submaximal exercise testing. A number of variables have been identified that could influence submaximal treadmill running performance, including circadian variation ${ }^{4}$, footwear ${ }^{4}$, training status ${ }^{4}$ and treadmill accommodation ${ }^{4}$.

Another important issue is the effect of training. Williams et al. ${ }^{5}$ and Morgan et al. ${ }^{4}$ addressed the question of training status in assessments of submaximal running economy in adult runners. These two studies indicated that the more highly trained the subject, the lower the variability between testing sessions. This led Morgan et al. ${ }^{4}$ to conclude that a stable measure of running economy could be obtained in a single data collection session involving trained, non-élite male runners if the testing environment was controlled to minimize non-biological variability.

The small number of reproducibility studies concerning children have focused mainly on the reliability and reproducibility of maximal oxygen consumption tests. For example, Cunningham et al. ${ }^{6}$ demonstrated a reliability coefficient of $76 \%$ for two maximal oxygen consumption tests within a 4 week period where the reliability coefficient represents the proportion of the total variability explained by betweensubject variability. This value is below the 0.90 value found in most adult studies, ${ }^{4,5}$.

No studies to date have assessed the stability of submaximal running performance in children. The primary aim of this study was to evaluate the variability of cardiorespiratory measurements at three submaximal exercise intensities in pre-pubertal children. For comparison we also measured the variability associated with maximal treadmill exercise performance. A secondary aim of the study was to predict the optimal number of visits that would be necessary to obtain stable submaximal and peak oxygen consumption values.

\section{Materials and methods}

Ten boys volunteered to take part in the study (mean(s.d.) age 10.7(0.71) years) (Table 1). Verbal assent to participate in the study was given by all the 
boys prior to the completion of informed consent forms by the parents/guardians of each subject; and the study was approved by the Ethics Committee of the Royal Hospital for Sick Children. All boys were pre-pubertal, based on parental reporting of the absence of voice change, no shaving and no pubic hair. Of the ten boys, five were rated as active and five very active on data obtained from a physical activity questionnaire.

All subjects underwent an habituation/accommodation visit before the testing ${ }^{7}$. At this visit all subjects were introduced to the treadmill with a short period of walking and running. In addition, the children were familiarized with all the equipment to be used in the cardiorespiratory testing.

\section{Measurement of cardiorespiratory parameters}

Expired gas measurements were made using a computerized metabolic cart (Sensormedics S2900Z, Bilthoven, The Netherlands). Measurement of cardiorespiratory variables were made every $20 \mathrm{~s}$ throughout the test collection period. The Sensormedics S2900Z uses a mass-flow anemometer for volume measurement. Pre-test calibration of the anemometer was achieved by the use of a 31 calibration syringe (Sensormedics, Bilthoven, The Netherlands). The $\mathrm{O}_{2}$ and $\mathrm{CO}_{2}$ analysers were calibrated by the introduction of two calibration gases $-26 \% \mathrm{O}_{2}, 0 \% \mathrm{CO}_{2}$, balance nitrogen and $16 \% \mathrm{O}_{2}, 4 \% \mathrm{CO}_{2}$, balance nitrogen - both immediately prior to, and just after the completion of each exercise test. Previous work has demonstrated the validity of the Sensormedics S2900Z metabolic cart in paediatric exercise testing ${ }^{8}$.

To facilitate the expired gas analysis and minimize dead space ventilation, all subjects were tested using paediatric mouthpieces and valves (Hans-Rudolph 1410B, dead space $16 \mathrm{ml}$, Hans-Rudolph Incorporated, Kansas City, USA). The differential pressure to the resistance of flow up to flow rates of $200 \mathrm{l} / \mathrm{min}$ was between $0.6-5.5 \mathrm{~cm} \mathrm{H}_{2} \mathrm{O}$ on the inspired side and $0.6-8.2 \mathrm{~cm} \mathrm{H}_{2} \mathrm{O}$ on the expired side. The valve and mouthpiece was supported by a head support (Hans-Rudolph, Model Number 1426). Small bore tubing assisted in minimizing dead space ventilation between the outflow of the valve and the entry port of the S2900Z metabolic cart.

Heart rate data were obtained by means of the PE3000 Sport Tester heart rate monitor (Polar Sports Ltd, Kempe, Finland). The treadmill (Power Jog M10, Cardinal Sports, Edinburgh, Scotland) speed was calibrated for each submaximal steady state stage. The testing was carried out in a laboratory with an environmental temperature between $21^{\circ} \mathrm{C}$ and $26^{\circ} \mathrm{C}$ and a relative humidity of between $43 \%$ and $54 \%$. In an attempt to control for possible circadian variations in submaximal running economy ${ }^{4}$, the time of testing remained constant for eight of the ten subjects (i.e. submaximal and peak $\mathrm{VO}_{2}$ tests were conducted in the afternoon). All subjects were advised to wear the same footwear each visit and all testing was completed within a 4 week period. A minimum of 3 days between each test was instigated in order to prevent any possible residual fatigue between sessions.

\section{Test protocols}

Submaximal economy runs

Two submaximal economy tests were administered within a 4 week period. The test consisted of three $6 \mathrm{~min}$ runs at $7.2,8.0$ and $8.8 \mathrm{~km} / \mathrm{h}$. Each $6 \mathrm{~min}$ run was preceded by a $2 \mathrm{~min}$ walk at $4.2 \mathrm{~km} / \mathrm{h}$ in order to introduce the subject to exercise at the onset and between submaximal exercise stages. It also provided an indication of any drift in baseline oxygen consumption as a result of external factors (temperature, fatigue and learning). In order to ensure that no residual effect existed between one submaximal economy stage and the next, the protocol was discontinuous in nature with passive recoveries of $8 \mathrm{~min}$ between each. On-line oxygen consumption measurements were taken throughout the $8 \mathrm{~min}$ submaximal stages using the Sensormedics S2900Z metabolic cart (Bilthoven, The Netherlands) and confirmed the return to baseline oxygen consumption. Based upon pilot work in this laboratory (Unnithan et al. 1992, unpublished observations) cardiorespiratory steady state was found to be achieved between 3 and $6 \mathrm{~min}$ within submaximal exercise testing. The average of the three $20 \mathrm{~s}$

Table 1. Demographic details of the boys studied

\begin{tabular}{|c|c|c|c|c|c|c|}
\hline \multirow[t]{2}{*}{ Subjects } & \multirow[t]{2}{*}{ Age (yrs) } & \multirow[t]{2}{*}{ Height $(\mathrm{cm})$} & \multicolumn{4}{|c|}{ Weight (kg) } \\
\hline & & & Visit 1 & Visit 2 & Visit 3 & Visit 4 \\
\hline 1 & 10.7 & 141 & 41.8 & 42.1 & 41.5 & 43.0 \\
\hline 2 & 9.5 & 133 & 28.6 & 29.0 & 29.0 & 28.7 \\
\hline 3 & 11.3 & 151 & 37.2 & 37.4 & 37.3 & 37.8 \\
\hline 4 & 11.8 & 143 & 31.4 & 31.6 & 31.4 & 31.7 \\
\hline 5 & 11.1 & 151 & 37.3 & 37.5 & 37.3 & 37.4 \\
\hline 6 & 10.5 & 145 & 35.3 & 35.9 & 35.5 & 35.3 \\
\hline 7 & 10.5 & 148 & 35.1 & 35.2 & 35.2 & 35.8 \\
\hline 8 & 9.9 & 136 & 29.0 & 28.7 & 29.5 & 29.5 \\
\hline 9 & 11.7 & 142 & 30.1 & 30.6 & 30.5 & 30.6 \\
\hline 10 & 10.3 & 138 & 31.5 & 31.6 & 31.6 & 31.5 \\
\hline Mean(s.d) & $10.7(0.71)$ & 142(5.79) & $33.7(4.08)$ & $34.0(4.13)$ & $33.9(3.89)$ & $34.1(4.28)$ \\
\hline
\end{tabular}


measurements made in the sixth and final minute was used for the subsequent analyses.

Peak $\mathrm{VO}_{2}$ test

Two peak $\mathrm{VO}_{2}$ tests were also administered within the 2-4 week period. The test protocol was a modified incremental test devised for paediatric testing 9 . After a 2 min warm-up walk at $4.2 \mathrm{~km} / \mathrm{h}$ and $0 \%$ gradient, the speed was then increased to $8.8 \mathrm{~km} / \mathrm{h}$ at $0 \%$ gradient and recording on the Sport Tester receivers and $\mathrm{S} 2900 \mathrm{Z}$ was initiated. Throughout the test the speed remained constant at $8.8 \mathrm{~km} / \mathrm{h}$, but the gradient was increased $2.5 \%$ every $2 \mathrm{~min}$ until volitional fatigue was achieved. The subjects were given extensive verbal encouragement to achieve their maximal exercising capacity. Attainment of peak $\mathrm{VO}_{2}$ was used as the maximal index ${ }^{10}$ and was judged to have been achieved when two of the three following criteria were attained: (1) heart rate within $10 \mathrm{bpm}$ of $200 \mathrm{bpm}$; (2) heart rate plateau (plateau was defined as less than a 5 beat increase from the penultimate to the final stage); (3) respiratory exchange ratio value of greater than 1.0. In addition, an estimation of the attainment of a plateau of $\mathrm{VO}_{2}$ values was conducted. The attainment of a plateau was considered to be the difference between the final $\mathrm{VO}_{2}$ value and the penultimate stage $\mathrm{VO}_{2}$ being less than the mean difference of the preceding stages. Practically, termination of the test occurred when, despite strong verbal encouragement from the researchers, the subject was unwilling or unable to continue. The sequence of testing was always a submaximal test followed by a peak test for all subjects. This sequence was repeated so that all subjects had two submaximal and two peak tests in total.

\section{Statistical methods}

Standard descriptive statistics (mean and s.d.) were used to summarize the data. A two way repeated measures analysis of variance statistics was used to test for visit and speed effects.

The coefficient of reliability (CR) was used to explore the reproducibility of cardiorespiratory measurements. After adjusting for significant visit, subject and speed effects the CR was calculated as follows:

$C R=\sigma^{2} B /\left(\sigma^{2} B+\sigma^{2} W\right)$

where $\sigma^{2} \mathrm{~B}=$ between subject variance and $\sigma^{2} \mathrm{~W}=$ within subject variance.

In any assessment of variability the major expectation is that variability between subjects dominates over the variability within subjects for any given dependent variable.

To provide the best estimate of the $C R$, the data were pooled across all three speeds for each boy. An hypothesis test was used to check that the same physiological trends existed across speeds for each boy, ensuring that this statistical manipulation was valid. All statistical calculations were performed using Minitab version 7.1 and a significance level of 0.05 was used.
Table 2. Mean(s.d.) values for cardiorespiratory variables at submaximal running speeds

\begin{tabular}{llll}
\hline Variable & $7.2(\mathrm{~km} / \mathrm{h})$ & $8.0(\mathrm{~km} / \mathrm{h})$ & $8.8(\mathrm{~km} / \mathrm{h})$ \\
\hline $\mathrm{VO}_{2}(\mathrm{ml} / \mathrm{kg} / \mathrm{min})$ & $35.2^{*}(2.59)$ & $38.5^{*}(2.65)$ & $40.7^{*}(3.53)$ \\
$\mathrm{Ve}(\mathrm{l} / \mathrm{min})$ & $32.2^{*}(3.8)$ & $34.5^{*}(2.84)$ & $36.5^{*}(3.73)$ \\
$\mathrm{HR}(\mathrm{beats} / \mathrm{min})$ & $165^{*}(20.9)$ & $176^{*}(18.9)$ & $185^{*}(16.2)$ \\
$\mathrm{RER}$ & $0.93(0.046)$ & $0.92(0.033)$ & $0.94(0.047)$ \\
\hline
\end{tabular}

All values are given as mean(s.d.)

${ }^{*}(P<0.05)$

Table 3. Mean(s.d.) values for cardiorespiratory variables at peak exercise intensity

\begin{tabular}{lll}
\hline Variable & Visit 1 & Visit 2 \\
\hline $\mathrm{VO}_{2}(\mathrm{ml} / \mathrm{kg} / \mathrm{min})$ & $58.8(6.48)$ & $59.6(4.18)$ \\
$\mathrm{Ve}(\mathrm{l} / \mathrm{min})$ & $63.4(10.1)$ & $63.6(7.8)$ \\
$\mathrm{HR}(\mathrm{beats} / \mathrm{min})$ & $200(10.0)$ & $205(4.9)$ \\
$\mathrm{RER}$ & $1.06(0.041)$ & $1.09(0.053)$ \\
\hline
\end{tabular}

All valves are given as mean(s.d.)

\section{Results}

\section{Submaximal running economy}

The mean change in body mass over the four testing periods was $0.57 \mathrm{~kg}$ (Table 1). Therefore, any variation in the submaximal energy cost of running was unlikely to be attributable to fluctuation in weight.

Four variables were measured across the three submaximal speeds and at maximal exertion: oxygen consumption, $\left(\mathrm{VO}_{2}, \mathrm{ml} / \mathrm{kg} / \mathrm{min}\right)$, ventilation (Ve, $1 / \mathrm{min})$, heart rate $(\mathrm{HR}, \mathrm{bpm})$ and respiratory exchange ratio (RER). For three of the variables there was a significant increase with increasing speed $\left(\mathrm{VO}_{2}, \mathrm{Ve}\right.$ and HR; Table 2$)$. RER was not significantly different with increasing speed. There was no evidence of a significant visit effect. Two variables, ventilatory equivalent for oxygen $\left(\mathrm{VeVO}_{2}\right)$ and fractional utilization (FU), measuring oxygen consumption at submaximal intensities as a percentage of the peak $\mathrm{VO}_{2}$, were measured only across the three submaximal running speeds. For both there were significant drops in levels between visits 1 and 2 $\left(\mathrm{VeVO}_{2}: 27.33\right.$ vs 26.56 and FU: 67.39 vs 64.01$)$ indicating a possible learning effect. FU exhibited the expected increase with speed confirming the boys used a higher proportion of their peak $\mathrm{VO}_{2}$ at each submaximal workload.

Based upon the data generated, it appeared that between- and within-subject variances differed at maximal compared to submaximal workloads for different indices. Consequently, $C R$ values were calculated separately. For submaximal speeds $\mathrm{VO}_{2}$ $\mathrm{HR}, \mathrm{VeVO}_{2}$ and $\mathrm{FU}$ were the most reliable, with between-subject variation accounting for over twothirds of the total variance. Ventilation and RER appeared only moderately reliable with between subject variation accounting for less than half the total variance (Table 4). 
Cardiorespiratory reproducibility during running in children: V. B. Unnithan et al.

Table 4. Between- and within-subject variance and coefficient of reliability values for pooled submaximal and maximal data

\begin{tabular}{lccc}
\hline Variable & $\sigma^{2} B$ & $\sigma^{2} W$ & Coefficient of reliability (\%) \\
\hline Submaximal & & & \\
$\mathrm{VO}_{2}(\mathrm{ml} / \mathrm{kg} / \mathrm{min})$ & 6.63 & 3.07 & 68 \\
$\mathrm{Ve}(\mathrm{lmin})$ & 5.1 & 5.2 & 50 \\
$\mathrm{RER}$ & 0.00075 & 0.0009 & 45 \\
$\mathrm{HR}(\mathrm{bpm})$ & 397.8 & 27.6 & 94 \\
$\mathrm{VeVO}$ & 5.19 & 1.76 & $75^{*}$ \\
$\mathrm{FU}(\%)$ & 68.4 & 15.5 & $82^{*}$ \\
Maximal & 19.20 & 10.39 & 65 \\
VO $(\mathrm{ml} / \mathrm{kg} / \mathrm{min})$ & 54.84 & 32.80 & 63 \\
RER Ve (l/min) & 0.000093 & 0.00154 & 38 \\
HR (bpm) & 15.9 & 50.2 & 24 \\
Time to exhaustion (min) & 1.298 & 0.747 & 63 \\
\hline
\end{tabular}

$\sigma^{2} \mathrm{~B}$, between subject variance; $\sigma^{2} \mathrm{~W}$, within subject variance; ${ }^{*}$ Significant visit effect, therefore adjusted means used in calculation of $C R$

Both an individual and an overall analysis were conducted for the submaximal running speeds. The overall analysis was undertaken to account for the particular variability of certain individual subjects. Since each speed involves fewer observations it was more likely to be affected by excessive random variability in a few subjects and hence distort the estimation of the CRs for the group. At increased submaximal running speeds, $H R$ and RER CR progressively increased. In contrast, ventilation with increasing running speed demonstrated a systematic decrease in the reliability coefficient. Submaximal oxygen consumption demonstrated no significant trend with increased treadmill running speed. However, the highest coefficient of reliability $(0.80)$ was noted at the first running speed $(7.2 \mathrm{~km} / \mathrm{h}$, Table 5 ). At the three submaximal running speeds the $C R$ for FU were $87.0 \%, 86.5 \%$ and $89.3 \%$ respectively.

Within-subject differences were investigated for ventilation, heart rate and oxygen consumption at all three running speeds. At $7.2 \mathrm{~km} / \mathrm{h}$, the group mean difference in $\mathrm{VO}_{2}$, expressed as a percentage of each individual's mean $\mathrm{VO}_{2}$ calculated from the two visits,

Table 5. Discrete analysis of coefficient of reliabilities at submaximal intensities

\begin{tabular}{lccc}
\hline & \multicolumn{3}{c}{ Speed $(\mathrm{km} / \mathrm{h})$} \\
\cline { 2 - 4 } Variable & 7.2 & 8.0 & 8.8 \\
\hline $\mathrm{VO}_{2}(\mathrm{ml} / \mathrm{kg} / \mathrm{min})$ & & & $68 \%$ \\
Ve $(\mathrm{l} / \mathrm{min})$ & $80 \%$ & $61 \%$ & $43 \%$ \\
$\mathrm{HR}(\mathrm{bpm})$ & $70 \%$ & $68 \%$ & $93 \%$ \\
RER & $69 \%$ & $79 \%$ & $78 \%$ \\
\hline
\end{tabular}

was $4.08 \%$. However, this moderate fluctuation obscured a wide range of intra-individual variation (range $=0.67 \%-7.99 \%$ ). At $8.0 \mathrm{~km} / \mathrm{h}$ the value was $5.61 \%$ (range $1.09 \%-11.28 \%$ ) and at $8.8 \mathrm{~km} / \mathrm{h}, 5.75 \%$ $(0.16 \%-17.26 \%)$. Similar values were generated for Ve and HR (Table 6).

Based upon the results in this study, the accuracy of the mean $\mathrm{VO}_{2}$ level of an individual derived from a two-test assessment would give a standard error of the mean (s.e.m.) of $\pm 1.25 \mathrm{ml} / \mathrm{kg} / \mathrm{min}$. The s.e.m. based upon a predicted four visits would be $\pm 0.9 \mathrm{ml} / \mathrm{kg} / \mathrm{min}$. This predicted value is derived from the standard error:

s.e.m. $=\frac{\sigma}{\sqrt{n}}$

where $\sigma=$ pooled submaximal standard deviation, $\sigma$ $=1.77$ and $n=$ target number of visits.

\section{Peak $\mathrm{VO}_{2}$ analysis}

At maximal exercise, $\mathrm{VO}_{2}$ ventilation and time to exhaustion appeared to be the most reliable responses; with between-person variability accounting for approximately two-thirds of the total variance (Tables 3 and 4). The mean $\Delta \mathrm{VO}_{2}$ expressed as a percentage of the mean $\mathrm{VO}_{2}$ from visits 1 to 2 was $5.3 \%$. However, this low intra-individual variation masked a high degree of between-subject variation (range $0.17-17.78 \%$ ). Results from the analysis of peak ventilation demonstrated a similar range $(\Delta \mathrm{Ve}$, mean $=10.27$, range $1.94 \%-19.48 \%)$. The same trend was noted with $\mathrm{HR}$ (mean $=3.15 \%$, range from $0 \%-14.14 \%)$.

All ten boys in the study achieved the criteria for peak $\mathrm{VO}_{2}$. In addition, three demonstrated plateaux

Table 6. Mean differences and range expressed as a percentage of mean value generated over two visits for $\mathrm{VO}, \mathrm{Ve}$ and $\mathrm{HR}$

\begin{tabular}{|c|c|c|c|c|c|c|}
\hline \multirow[b]{2}{*}{ Variable } & \multicolumn{2}{|l|}{$7.2 \mathrm{~km} / \mathrm{h}$} & \multicolumn{2}{|l|}{$8.0 \mathrm{~km} / \mathrm{h}$} & \multicolumn{2}{|l|}{$8.8 \mathrm{~km} / \mathrm{h}$} \\
\hline & Mean difference & Range & Mean difference & Range & Mean difference & Range \\
\hline $\mathrm{VO}_{2}(\%)$ & 4.08 & $0.67-0.99$ & 5.61 & $1.09-11.28$ & 5.75 & $0.16-17.26$ \\
\hline $\operatorname{Ve}(\%)$ & 9.07 & $1.45-20.29$ & 6.17 & 1.04-20.09 & 3.74 & $0.95-5.49$ \\
\hline HR (\%) & 8.42 & $0-25.53$ & 5.73 & $0-17.50$ & 3.74 & $0.95-5.49$ \\
\hline
\end{tabular}


at both peak $\mathrm{VO}_{2}$ tests. However, $\Delta \mathrm{VO}_{2}$ expressed as a percentage of the mean $\mathrm{VO}_{2}$ was low in one individual $(0.58 \%)$ and high in the other two $(11.63 \%$ and $12.2 \%$ ). Of the remaining seven boys, six demonstrated a plateau of peak $\mathrm{VO}_{2}$ at one of the two visits. Their $\Delta \mathrm{VO}_{2}$ values expressed as a percentage of the mean $\mathrm{VO}_{2}$ was $4.97 \%$ (range $0.17 \%-17.78 \%$ ). One subject failed to attain a plateau at either of the two visits, attaining a value of $0.79 \%$. Therefore, there appeared to be no significant pattern linking the presence or absence of the $\mathrm{VO}_{2}$ plateau with the test-retest reliability within an individual.

Within this testing environment a two visit assessment of an individual was capable of estimating the mean peak $\mathrm{VO}_{2}$ with a standard error of the mean of $\pm 2.28 \mathrm{ml} / \mathrm{kg} / \mathrm{min}$. To achieve an estimate of mean peak $\mathrm{VO}_{2}$ with a s.e.m. of $\pm 0.5 \mathrm{ml} / \mathrm{kg} / \mathrm{min}$ would require a predicted 41 peak $\mathrm{VO}_{2}$ assessments. The calculation of the number of predicted visits required for both submaximal and peak $\mathrm{VO}_{2}$ testing does not include the necessary visit for habituation and accommodation that should precede testing.

\section{Discussion}

Results from the submaximal running performance analysis demonstrated that $\mathrm{VO}_{2}, \mathrm{HR}, \mathrm{VeVO}_{2}$ and $\mathrm{FU}$ were the most reliable submaximal parameters, accounting for over $67 \%$ of the total variance. Ventilation and RER were only moderately reliable, accounting for less than half of the total variation. At maximal exercise, $\mathrm{VO}_{2}$, ventilation and time to exhaustion appeared most reliable, both accounting for over $67 \%$ of the total variance. Between-subject variability dominated in the assessment of these variables, accounting for approximately $67 \%$ of the total variance.

In many adult studies, CRs of the order of $90 \%$ have been generated for submaximal oxygen consumption. It would be hoped that the variability between individuals would dominate over that seen within individuals. In adults the ratio of between- to within-subject variability is $9: 1$. In the present study the ratio of between- to within-subject variability was approximately 2:1.

One possible reason for this difference was the homogeneity of the group being tested. The smaller inter-individual distribution of physiological data resulted in a lower between subject variance. Consequently, the subsequent estimations of CRs are likely to be underestimated (assuming within-subject variability stays the same) because of the homogeneous nature of the subject population. In a wider fitness spectrum the between-subject variability would have been larger and again assuming that within-subject variability remained constant this would have given rise to estimations of CR which would be higher. Accordingly, our conclusions of the reliability of repeated submaximal and maximal exercise testing can only be applied to fairly homogeneous childhood populations. This highlights the need for each laboratory to determine the number of test sessions specific to the nature of their particular population. A number of other factors may also have contributed to the lower reliability of this sample. A significant visit effect was noted for $\mathrm{VeVO}_{2}$. The pattern of breathing was more efficient at the second visit, possibly reflecting greater familiarity with the test apparatus. This suggests that despite careful and extensive efforts, a degree of accommodation was occurring during the test procedures. Frost et al. ${ }^{11}$ demonstrated that there was no set pattern to the number of treadmill visits required to achieve accommodation in a paediatric population. While it is true that children have a low tolerance of extreme heat, in neutral or moderately warm climates they can thermoregulate when exercising ${ }^{12}$ as effectively as adults. Consequently, the rather wide temperature range of the laboratory would be unlikely to affect performance.

Submaximal and maximal running performance is the product of both physiological and psychological interaction $^{13}$. The contribution of motivation towards physiological variation was not measured in this study. However, in an attempt to control this variable, all the boys were motivated in a similar manner throughout all the testing sessions.

In this study there was no pattern in the CRs for $\mathrm{VO}_{2}$ with increased treadmill running speed. These findings are consistent with the data of Morgan et al. ${ }^{3}$ Armstrong and Costill ${ }^{1}$ also demonstrated that certain submaximal treadmill speeds (Table 5) represented 'inefficient' workloads for their specific population. It is possible that all the submaximal treadmill speeds selected for the boys in this study represented relatively inefficient running speeds and that this biomechanical inefficiency contributed toward the lack of pattern in $\mathrm{VO}_{2}$ reliability.

Another factor capable of mediating submaximal running performance is training status. The influence of training status on $\mathrm{VO}_{2}$ variability was considered by Katch et al. ${ }^{14}$ and Williams et al. ${ }^{5}$ These authors hypothesized that biological variation would be larger in untrained subjects due to the greater variation in both the transport and extraction of oxygen at the cellular level. The group of boys used in this study could be classed as active, but not highly trained. Therefore, the greater variability compared to adult studies could arise as a product of their differing metabolic profiles during exercise or as a consequence of their lack of training. In keeping with the latter, Williams et al. ${ }^{5}$ calculated higher estimates of variability than those obtained by Morgan et al. ${ }^{4}$, who had used well-trained runners.

In this study there was no evidence to link the presence (nine boys) or absence (one boy) of a plateau in $\mathrm{VO}_{2}$ with the reliability of peak $\mathrm{VO}_{2}$. This contradicts the data derived by Cunningham et al. ${ }^{15}$, who traced a greater reliability in peak $\mathrm{VO}_{2}$ to those subjects who reached a plateau and attributed this to those boys being more capable of generating energy from anaerobic sources. The capacity to derive energy from anaerobic sources would allow sustained work output at peak aerobic power and, consequently, greater reliability in the peak aerobic value.

Results from the present analysis of submaximal and maximal $\mathrm{VO}_{2}$ reliability also indicated that the degree of reliability was not related to the exercise intensity (Tables 4 and 5). There is conflicting evidence about the impact of exercise intensity upon 
the CR. Armstrong and Costill ${ }^{1}$ demonstrated that within-subject day-to-day variation was lower at submaximal rather than maximal levels while Taylor ${ }^{16}$ reported the opposite. The possible psychological implications of attaining peak exercise intensities could have contributed to the differences between the two populations.

Two further cardiorespiratory variables were investigated in order to assess their suitability as indices of stability: ventilation and heart rate. Ventilatory data generated from this study are in agreement with that generated by Armstrong and Costill ${ }^{1}$ and Davies et al. ${ }^{17}$ Higher CRs were obtained at maximal compared to submaximal exercise. Davies et al. ${ }^{17}$ hypothesized that an increased muscle mass recruitment at maximal exercise, resulting in altered proprioceptive reflexes from joints and muscles, led to more stable ventilation patterns at maximal exercise. However, in absolute terms, the magnitude of the CRs was low at both submaximal and maximal workloads, in agreement with Taylor ${ }^{14}$. RER demonstrated low CR at both maximal $(38 \%)$ and submaximal exercise $(45 \%)$ intensities, reflecting the multitude of substrate and ventilatory factors that influence this measurement. Consequently, neither Ve nor RER should be be regarded as reliable indices.

The lack of change in submaximal heart rate from visits 1 and 2 (CR, 94\%) implies that no training effect has occurred in the cirulatory system at submaximal exercise. Davies et al. ${ }^{15}$ demonstrated a decreased submaximal heart rate for constant $\mathrm{VO}_{2}$ with repeated testing mediated perhaps through adjustments in stroke volume and/or $\mathrm{O}_{2}$ extraction at the cellular level. This would not appear to be the case with the submaximal heart rate profiles obtained in this study. The short duration of the project (4 weeks), effectively controlled any training effects that may have been present. However, the small number of maximal heart rate recordings obtained made any conclusions tentative.

The high CR obtained for fractional utilization $82 \%$ - and at the three submaximal speeds $(87.0 \%$, $86.5 \%$ and $89.3 \%$ ) have significant implications. Exercise intensities are prescribed for both clinical and sporting assessments based upon treadmill testing. Therefore, the data from this study indicates that prescribing an exercising range relative to a percentage of peak $\mathrm{VO}_{2}$ is a reasonably robust approach within a laboratory setting. The visit effect, while statistically significant, has minimal implications in the prescription of exercise intensities for laboratory based training and rehabilitation.

The aim of this study was to quantify the degree of reproducibility that exists with submaximal and maximal exercise testing, and to predict the optimal and most practical number of visits that would be necessary to achieve stable physiological data. At the submaximal level, to achieve an estimate of submaximal $\mathrm{VO}_{2}$ with a standard error of $\pm 0.9 \mathrm{ml} / \mathrm{kg} / \mathrm{min}$, four submaximal tests would be required for a given individual. This would be an impractical and unrealistic test schedule for most children. If the same rationale is applied to peak $\mathrm{VO}_{2}$ testing, to achieve an estimate of mean peak $\mathrm{VO}_{2}$ with a standard error of $\pm 0.5 \mathrm{ml} / \mathrm{kg} / \mathrm{min}$, 41 peak $\mathrm{VO}_{2}$ tests would be re- quired. Standard errors of these magnitudes were arbitrary selections. However, based upon published data $^{11}$, they reflect the most stringent levels of measurement accuracy that have been employed for the assessment of submaximal and peak oxygen consumption.

In conclusion, single economy testing sessions are valid for estimating group stability of running economy in normally active boys. If individual profiles are required, multiple submaximal and maximal testing will be necessary.

Daniels ${ }^{2}$ stated 'Even when controlling for the multiple external factors that influence running economy (circadian variation, footwear, training and length of treadmill accommodation), significant differences still exist in the aerobic demands of running between and within well trained runners'. The need for a multidisciplinary approach incorporating metabolic, structural and mechanical factors to explain fully within-subject variation in paediatric running economy is clear.

\section{References}

1 Armstrong LE, Costill DL. Variability of respiration and metabolism: responses to submaximal cycling and running. Res Q Exerc Sport 1985; 56: 93-6.

2 Daniels J, Scardina $\mathrm{N}$, Hayes J, Foley $\mathrm{P}$. Variations in $\mathrm{VO}_{2}$ submax during treadmill running. Med Sci Sports Exerc 1984; 16: 108.

3 Morgan DW, Baldini FD, Martin PE. Day to day stability in running economy and step length among well-trained male runners. Int Sports Med 1987; 8: 242

4 Morgan DW, Martin PE, Krahenbuhl GS, Baldini FD. Variability in running economy and mechanics among trained male runners. Med Sci Sports Exerc 1991; 23: 378-83.

5 Williams TJ, Krahenbuhl GS, Morgan DW. Daily variation in running economy of moderately trained male runners. Med Sci Sports Exerc 1991; 23: 944-8.

6 Cunningham DA, Telford D, Swart GT. The cardiopulmonary capacities of young hockey players age 10. Med Sci Sports Exerc 1976; 8: 23-5.

7 Shephard RJ. Tests of maximal oxygen uptake - a critical view. Sports Med 1984; 1: 99-124.

8 Unnithan VB, Wilson J, Aitchison TC, Paton JY. Validation of microprocessor controlled exercise testing system (Sensormedics S2900Z) in children. In: Coudert J, Van Praagh E, eds. Children and Exercise XVI Paediatric Work Physiology, 51.

9 Unnithan VB, Eston RG. Stride frequency and submaximal running economy in adults and children. Paediatric Exercise Science 1990; 2: 149-55.

10 Williams JR, Armstrong N. The influence of age and sexual maturation on children's blood lactate response to exercise. Paediatric Exercise Science 1991; 3: 111-20.

11 Frost G, Bar-Or O, Dowling J, White C. Habituation of children to treadmill walking or running: metabolic and kinematic analysis. Med Sci Sports Exerc 1993; 25(Suppl): 8.

12 Bar-Or O. Pediatric Sports Medicine for the Practitioner. New York, USA: Springer-Verlag, 1983.

13 Crews DJ. Psychological state of running economy. Med Sci Sports Exerc 1992; 24: 475-82.

14 Katch VL, Sady SS, Freedson P. Biological variability in maximum aerobic power. Med Sci Sports Exercise 1982; 14: 21-5.

15 Cunningham DA, MacFarlane Van Waterschoot B, Paterson $\mathrm{DH}$, Lefcoe M, Sangal SP. Reliability and reproducibility of maximal oxygen uptake measurement in children. Med Sci Sports and Exerc 1977; 9: 104-8.

16 Taylor C. Some properties of maximal and submaximal exercise with reference to physiological variation and the measurement of exercise tolerance. Am J Phys 1944; 144: 200-12.

17 Davies CTM, Tuxworth W, Young JM. Physiological effects of repeated exercise. Clin Sci 1970; 39: 247-58. 